Louisiana State University

LSU Digital Commons

Faculty Publications

Department of Physics \& Astronomy

7-13-2006

\title{
Attosecond pulse trains generated using two color laser fields
}

\author{
J. Mauritsson \\ Louisiana State University \\ P. Johnsson \\ Lunds Tekniska Högskola \\ E. Gustafsson \\ Lunds Tekniska Högskola
}

A. L'Huillier

Lunds Tekniska Högskola

K. J. Schafer

Louisiana State University

See next page for additional authors

Follow this and additional works at: https://digitalcommons.Isu.edu/physics_astronomy_pubs

\section{Recommended Citation}

Mauritsson, J., Johnsson, P., Gustafsson, E., L'Huillier, A., Schafer, K., \& Gaarde, M. (2006). Attosecond pulse trains generated using two color laser fields. Physical Review Letters, 97 (1) https://doi.org/ 10.1103/PhysRevLett.97.013001

This Article is brought to you for free and open access by the Department of Physics \& Astronomy at LSU Digital Commons. It has been accepted for inclusion in Faculty Publications by an authorized administrator of LSU Digital Commons. For more information, please contact ir@lsu.edu. 


\section{Authors}

J. Mauritsson, P. Johnsson, E. Gustafsson, A. L'Huillier, K. J. Schafer, and M. B. Gaarde 


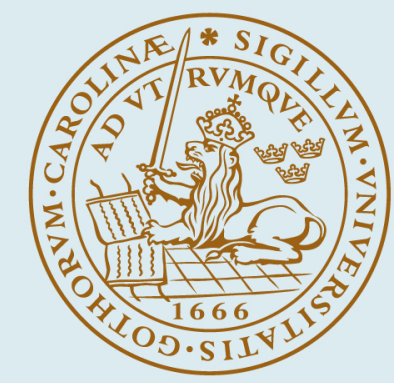

\section{LUND UNIVERSITY}

\section{Attosecond pulse trains generated using two color laser fields}

Mauritsson, Johan; Johnsson, Per; Mansten, Erik; L'Huillier, Anne; Schafer, K. J.; Gaarde, M. B.

Published in:

Physical Review Letters

DOI:

10.1103/PhysRevLett.97.013001

2006

Link to publication

Citation for published version (APA):

Mauritsson, J., Johnsson, P., Mansten, E., L'Huillier, A., Schafer, K. J., \& Gaarde, M. B. (2006). Attosecond pulse trains generated using two color laser fields. Physical Review Letters, 97(1), [013001]. https://doi.org/10.1103/PhysRevLett.97.013001

\section{Total number of authors:}

6

\footnotetext{
General rights

Unless other specific re-use rights are stated the following general rights apply:

Copyright and moral rights for the publications made accessible in the public portal are retained by the authors and/or other copyright owners and it is a condition of accessing publications that users recognise and abide by the legal requirements associated with these rights.

- Users may download and print one copy of any publication from the public portal for the purpose of private study or research.

- You may not further distribute the material or use it for any profit-making activity or commercial gain

- You may freely distribute the URL identifying the publication in the public portal

Read more about Creative commons licenses: https://creativecommons.org/licenses/

Take down policy

If you believe that this document breaches copyright please contact us providing details, and we will remove

access to the work immediately and investigate your claim.
} 


\title{
Attosecond Pulse Trains Generated Using Two Color Laser Fields
}

\author{
J. Mauritsson, ${ }^{1,2}$ P. Johnsson, ${ }^{2}$ E. Gustafsson, ${ }^{2}$ A. L'Huillier, ${ }^{2}$ K. J. Schafer, ${ }^{1}$ and M. B. Gaarde ${ }^{1}$ \\ ${ }^{1}$ Department of Physics and Astronomy, Louisiana State University, Baton Rouge, Louisiana 70803-4001, USA \\ ${ }^{2}$ Department of Physics, Lund Institute of Technology, P.O. Box 118, SE-221 00 Lund, Sweden
}

(Received 21 February 2006; published 5 July 2006)

\begin{abstract}
We investigate the spectral and temporal structure of high harmonic emission from argon exposed to an infrared laser field and its second harmonic. For a wide range of generating conditions, trains of attosecond pulses with only one pulse per infrared cycle are generated. The synchronization necessary for producing such trains ensures that they have a stable pulse-to-pulse carrier envelope phase, unlike trains generated from one color fields, which have two pulses per cycle and a $\pi$ phase shift between consecutive pulses. Our experiment extends the generation of phase stabilized few cycle pulses to the extreme ultraviolet regime.
\end{abstract}

PACS numbers: $32.80 . \mathrm{Rm}, 32.80 . \mathrm{Qk}, 42.65 . \mathrm{Ky}$

The ability to stabilize femtosecond optical frequency combs in the visible and near infrared wavelength regions has lead to a variety of new applications, ranging from precision spectroscopy to attophysics [1-4]. This achievement provides a connection between high precision measurements and ultrafast laser science, since stabilizing the frequency comb also enables control of the carrier envelope phase (CEP) [5]. This phase has significant implications when few cycle pulses are used [6,7], and phase stabilized few cycle pulses have been used in a number of novel ionization studies [8,9]. In this Letter we extend the concept of phase stabilized few cycle pulses to the extreme ultraviolet (XUV) regime by generating a train of attosecond pulses with the same CEP from pulse to pulse in the train.

Our pulses are synthesized by selecting a number of synchronized high harmonics generated in argon by an intense infrared (IR) laser and its second harmonic. As is well known, a one color driving field yields a spectrum of odd harmonics due to inversion symmetry. When several of these harmonics are synchronized, they give rise to an attosecond pulse train (APT) with two pulses per cycle [10-12]. In this experiment, we use a two color driving field $\mathcal{E}(t)=A \sin \omega_{1} t+B \sin \left(2 \omega_{1} t+\varphi\right)$, where $A$ and $B$ are amplitudes, $\omega_{1}$ is the IR frequency, and $\varphi$ is a controllable phase difference. This breaks the inversion symmetry and produces a spectrum containing both even and odd harmonics [13-15]. We directly measure the time structure of the emitted radiation and show that for a wide range of $\varphi$ just one pulse per IR cycle is generated. Our theoretical calculations show that this result is a single atom effect which is very general and robust. Furthermore, the presence of just one pulse per cycle may be taken as evidence that consecutive pulses in the train have the same CEP.

This conclusion is most easily understood via the close analogy between phase locked high harmonics and optical frequency combs. A one color driving field results in a mode spacing of $\omega_{\text {rep }}=2 \omega_{1}$, and the time between two pulses is then half the period of the driving field $\left(T_{1} / 2\right)$.
The difference in CEP between consecutive pulses, $\Delta \phi$, is related to the frequency offset, $\delta$, of the modes in the frequency comb with respect to integer multiples of $\omega_{\text {rep }}$, through $2 \pi \delta=\omega_{\text {rep }} \Delta \phi$ [5]. Since only the odd harmonics are included, $\delta=\omega_{1}$, which leads to $\Delta \phi=\pi$ and a phase flip from pulse to pulse in the train [Figs. 1(a) and 1(b)]. With a two color driving field, the mode spacing is $\omega_{\text {rep }}=$ $\omega_{1}$ and the harmonics are positioned at integer multiples of $\omega_{\text {rep }}$ so that the frequency offset $\delta$ is equal to 0 , thereby avoiding the pulse-to-pulse phase flip [Figs. 1(c) and 1(d)].

In the experiment we use a two color Michelson interferometer to generate, separate, and control three pulses: two IR pulses (pump and probe) and a frequency doubled pulse (blue). The pump and the blue pulses are combined to generate the APT, and the probe is used to measure its temporal structure. Incoming $1.6 \mathrm{~mJ}, 800 \mathrm{~nm}$ laser pulses are first frequency doubled in a $1.3 \mathrm{~mm}$ thick KDP (potas-
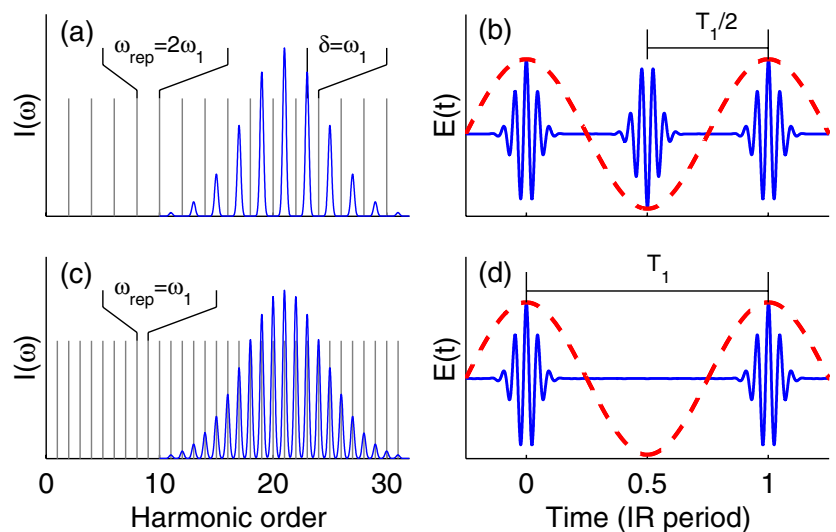

FIG. 1 (color online). (a) A one color driving field results in a frequency offset of $\delta=\omega_{1}$. (b) Phase locking produces an APT (solid line) with periodicity equal to half that of the driving field (dashed line) and a $\pi$ phase shift between consecutive pulses. (c) A two color field gives $\delta=0$. (d) Phase locking yields an APT with periodicity equal to that of the driving field, and a stable carrier envelope phase. 
sium dihydrogen phosphate), type I crystal. The second harmonic is separated from the IR using a dichroic beam splitter and sent into one arm of the interferometer. The remaining IR light is split into equally strong pump and probe beams, and the pump is recombined with the blue beam. We change the relative phase difference, $\varphi$, between the pump and the blue pulses using a rotatable $100 \mu \mathrm{m}$ thick delay plate. A half wave plate in the blue arm of the interferometer ensures that the IR pump and the blue pulses have the same polarization. Harmonics are generated by focusing $(f / 40)$ the two color pulses into a $3 \mathrm{~mm}$ long windowless cell filled with 40 mbar of argon. We estimate their intensities to be approximately $2 \times 10^{14} \mathrm{~W} / \mathrm{cm}^{2}$ (IR) and $1 \times 10^{14} \mathrm{~W} / \mathrm{cm}^{2}$ (blue) in the focus, which is located a few $\mathrm{mm}$ before the entrance to the cell (this leads to an IR:blue intensity ratio of $\approx 6-7$ in the center of the cell). The output XUV light is spatially and spectrally filtered using a hard aperture and a $200 \mathrm{~nm}$ thick aluminum foil, to remove harmonics below the 11 th $[12,16]$.

The harmonic radiation is spectrally characterized by photoionizing argon atoms and recording the electron spectra. A toroidal mirror is used to focus the radiation into a magnetic bottle electron spectrometer (MBES) with an argon background pressure of $2 \times 10^{-4}$ mbar. The MBES collects electrons emitted within a $2 \pi$ solid angle around the polarization direction of the XUV. The number of detected electrons per shot is kept low to avoid space charge effects and the spectra are measured over 30000 acquisitions. The photoelectron spectra in Fig. 2 show that both the total yield and the overall spectral structure of the harmonics change with $\varphi$, whereas the position of the individual harmonics remains the same. We find that for the value of $\varphi$ that corresponds to the maximum generation efficiency, the overall spectral structure is smooth with odd and even harmonics of comparable strengths (left inset of

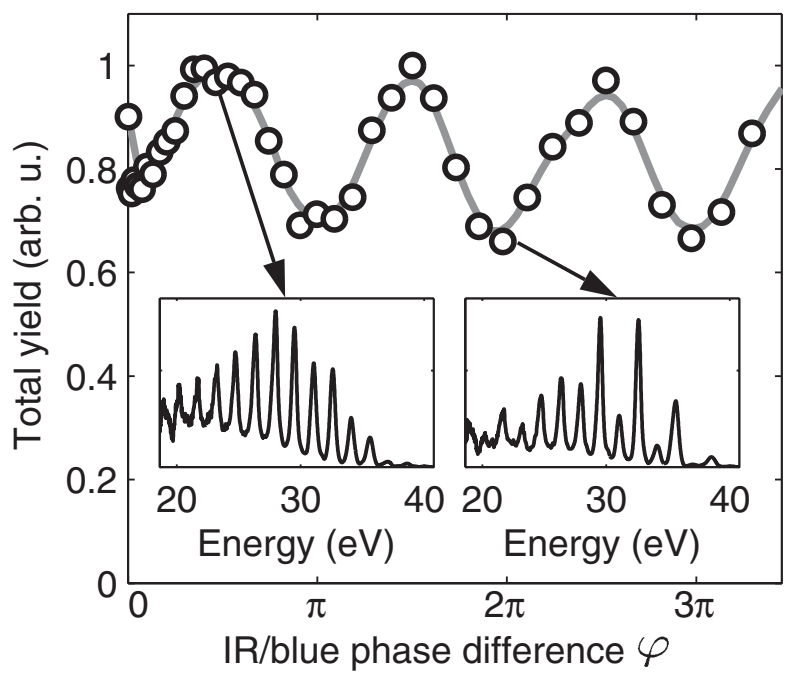

FIG. 2. Modulation in the total harmonic yield as a function of $\varphi$. The insets show the harmonic spectra for maximum (left) and minimum (right) yields.
Fig. 2), whereas the generation efficiency decreases and the structure becomes irregular when $\varphi$ is shifted by $\pi / 2$ (right inset).

The temporal structure of the generated APTs is studied by photoionizing argon atoms in the presence of the IR probe field and recording the electron spectra as a function of the XUV/probe delay. The technique used is similar to the attosecond streak camera proposed in [17], with the difference that here we collect electrons over a $2 \pi$ solid angle. The two beams are collinear, have the same polarization, and are focused by the same toroidal mirror. With this configuration, a significant amount of momentum is imparted to the photoelectrons by the probe. The momentum exchange is given by $\Delta \mathbf{p}=-e \mathbf{A}(t)$, where $\mathbf{A}(t)$ is the vector potential of the probe field and $-e$ the charge of the electron. The short duration of the attosecond pulses allows us to precisely control the instant of ionization, and the momentum exchange is therefore well defined as a function of the delay between the attosecond and the probe pulses. The largest momentum exchange occurs when the ionization time coincides with a peak in the vector potential of the probe field. The electron will either gain or lose energy, depending on the sign of the vector potential and the initial direction of the electron. In this way we get a one-to-one correspondence between the highest energy gain and the peaks in the APT envelope [16]. In Fig. 3(a) the measured photoelectron spectra as a function of XUV/ probe delay is shown for the value of the IR/blue phase difference $\varphi$ that gives the maximum harmonic yield. The periodicity of the high energy part of the spectrum clearly indicates that the APT has only one pulse per IR cycle (2.6 fs).

In order to compare the overall temporal structures of different APTs, we integrate the high energy portions of the spectra where there are very few electrons without the probe field (above $18.3 \mathrm{eV}$ ) [18]. The results are presented in Figs. 3(b)-3(e). The time profile shown in (b) is derived from the data shown in (a) with one pulse per IR cycle. In (c) we have changed $\varphi$ by $\pi / 4$ and the time profile now exhibits two pulses per IR cycle with a spacing which is not half an IR cycle. This phase corresponds to the minimum harmonic yield; see Fig. 2. In (d), where $\varphi$ is shifted by an additional $\pi / 4$, the temporal structure of the APT is again one pulse per cycle, but shifted by half a cycle compared to (a). For comparison, in Fig. 3(e) we show the time profile of an APT generated by the IR pump alone, with a clear half-cycle periodicity.

Measuring the APT time structure for many different values of $\varphi$, we find that the synchronization leading to the generation of one pulse per IR cycle is a robust effect. We see an indication of a more complicated time structure [as in Fig. 3(c)] for only a small range of $\varphi$ around the values corresponding to the minima in the harmonic yield. Our results demonstrate that the use of two color driving fields is a reliable method for generating trains of attosecond 

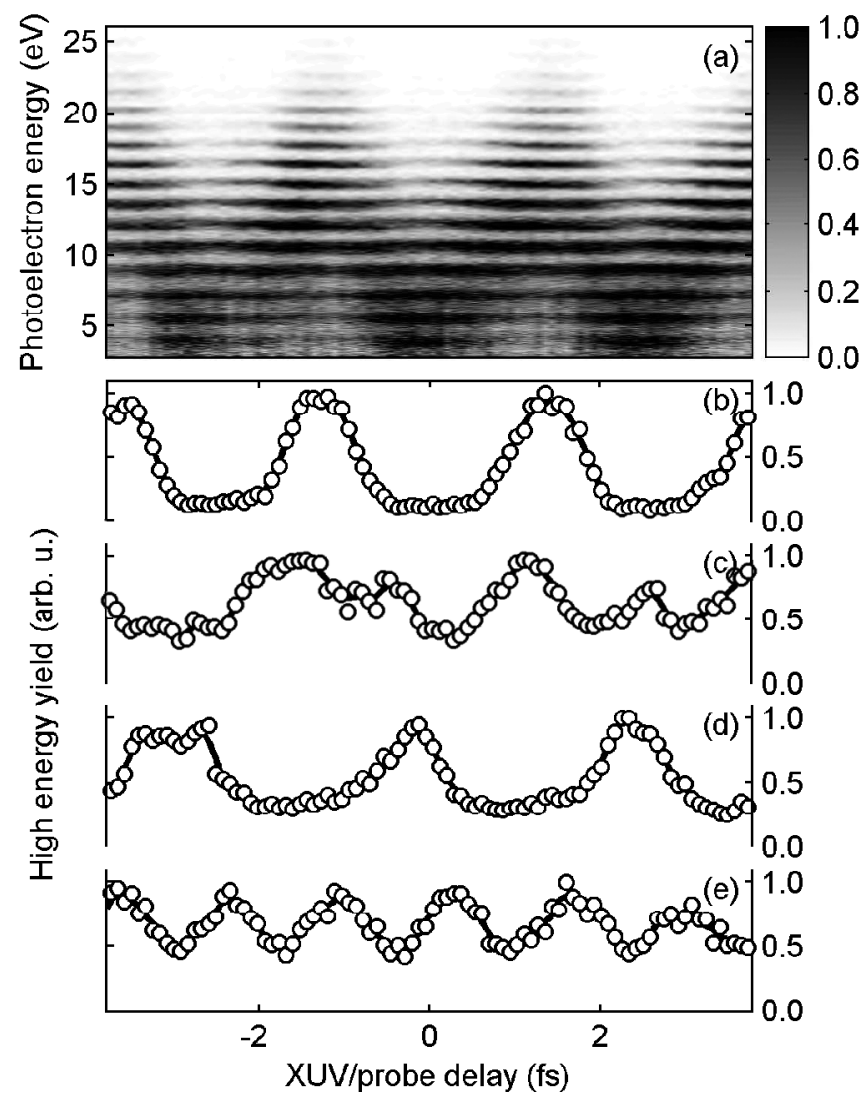

FIG. 3. (a) Photoelectron spectra versus delay between APT generated with a two color field and IR probe. The periodicity is one IR cycle. (b)-(e) Integrated electron yield above $18.3 \mathrm{eV}$ for four different APTs. (b) $\varphi=0$; (c) $\varphi=\pi / 2$; (d) $\varphi=\pi$. In (e) an APT generated with only the IR pump pulse is shown.

pulses that are free of the $\pi$ pulse-to-pulse phase difference inherent in APTs generated with one color driving lasers, as we further discuss below.

Our theoretical calculations reveal that the synchronization of odd and even harmonics observed in the experiment is a result of the single atom response to the two color field. An example of a two color electric field is shown in Fig. 4(a) (black line) for a IR/blue intensity ratio of two, and a phase difference of $\varphi=0.7 \pi$. The shape and strength of the field in consecutive half-cycles differ beyond the simple sign change that occurs in a one color field. This increased complexity means that there are two different and interwoven evolutions of the electric field over one IR cycle, one characterized by a half-cycle in which the electric field is strong, followed by half-cycle in which it is weak (we will refer to this as high $\rightarrow$ low), and vice versa (low $\rightarrow$ high). The two different electric field evolutions give rise to different and separate continuum dynamics, each generating a train of XUV pulses with a periodicity of one IR cycle. These two trains are offset from each other, and so in general we expect to find more than one pulse per IR cycle.
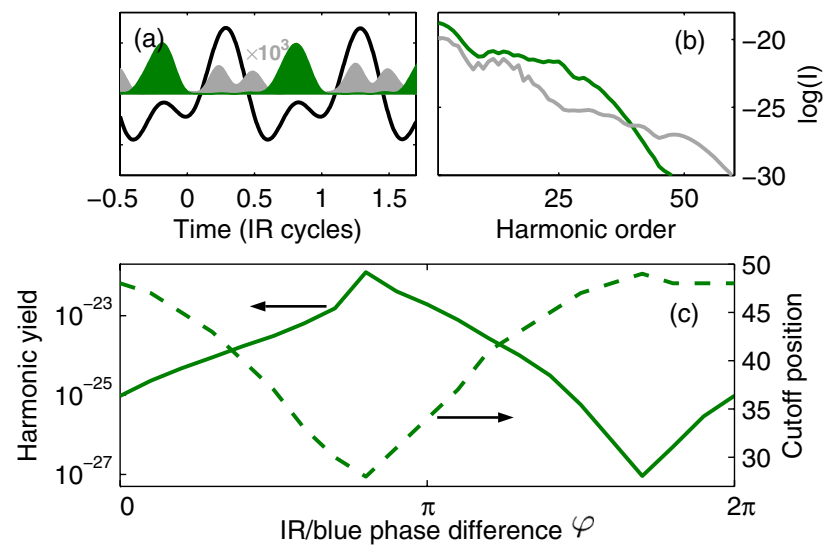

FIG. 4 (color online). Calculated harmonic generation when the ionization is restricted to occur only when the electric field is positive (thick green line) or negative (thin gray line). The intensities are $2 \times 10^{14} \mathrm{~W} / \mathrm{cm}^{2}$ (IR) and $1 \times 10^{14} \mathrm{~W} / \mathrm{cm}^{2}$ (blue), and $\varphi$ is $0.7 \pi$ in (a) and (b). (a) The two color electric field together with the temporal structure of the generated APT. (b) The corresponding spectra for ionization when the field is positive or negative. (c) Cutoff position and yield as a function of $\varphi$ when the ionization is restricted to positive fields. The results for the negative half-cycles are identical except for a translation by $\pi$.

For the electric field shown in Fig. 4(a), we show in Fig. 4(b) the two separate contributions to the full harmonic spectrum from the two electric field evolutions, calculated within the strong field approximation (SFA) [19]. The thick green curve in Fig. 4(b) shows the contribution from the high $\rightarrow$ low evolution alone. This has been calculated by restricting the ionization step in the SFA to occur only when the electric field is positive. The thin gray curve shows the contribution from the low $\rightarrow$ high evolution alone, calculated by restricting the ionization to occur only when the electric field is negative. The two spectra are very different: one has a larger yield and a lower cutoff energy than the other. Figure 4(c) shows that this inverse relation between the cutoff energy and the yield at the cutoff extends over the whole range of $\varphi$. The reason for this is that, in general, the harmonic yield is controlled by the electric field strength at the time of tunneling, whereas the cutoff energy is determined by the electric field that accelerates the electrons back toward the ion core a halfcycle later [19]. In the two color field, the symmetry between the ionizing and the reaccelerating field is broken, which explains the difference between the two sets of continuum dynamics in Fig. 4(b).

Similar to the harmonic spectrum, the time profile of an APT synthesized from several harmonics will also have two contributions. In Fig. 4(a) the time profiles of APTs synthesized from harmonics around the lower energy, higher yield cutoff in Fig. 4(b) $\left(\sim 25 \omega_{1}\right)$ are shown. The time profiles due to the high $\rightarrow$ low (low $\rightarrow$ high) electric field evolution are shown separately in dark green (light 
gray). We note that the gray curve, which has been multiplied by 1000, shows multiple peaks because these harmonics belong to the plateau region of the low $\rightarrow$ high spectrum [20]. An APT with effectively only one pulse per IR cycle can therefore be synthesized by combining a number of harmonics near the low energy, high yield cutoff, because the high $\rightarrow$ low train (green) is so much stronger than the low $\rightarrow$ high train (gray).

By calculating the full harmonic spectrum as a function of $\varphi$, we find that the method outlined above for the specific case of $\varphi=0.7 \pi$ is very robust. For almost all values of $\varphi$ there are two cutoffs: a high yield, low energy one, and a low yield, high energy one. By selecting harmonics near the low energy cutoff, as was done in the experiment, a single pulse per IR cycle can be generated. For a small range of $\varphi$, the harmonic generation is equally efficient for the two different field evolutions and we find two pulses per IR cycle. These values of $\varphi$ correspond to the lowest total harmonic yield, confirming the experimental finding that the APTs with one pulse per cycle correspond to the stronger harmonic yield.

Our calculations show that the single atom phase locking is robust with respect to the IR intensity and the IR/blue intensity ratio, as long as the blue intensity is more than $\sim 10 \%$ of the IR intensity. We have also compared the SFA results to numerical integration of the time-dependent Schrödinger equation within the single active electron approximation [21] and find that the calculations agree well for harmonics in the end of plateau and cutoff region. Finally, we have performed calculations including the macroscopic response of the argon gas by numerically solving the wave equation in the slowly evolving wave approximation [4], using the SFA to calculate the atomic response [22]. The synchronization is preserved after propagation through the gas, as is its dependence on $\varphi$.

In addition, our calculations show that the effect of the temporal intensity variation of the driving laser, which may induce a slow variation in the CEP of the attosecond pulses within the train [23] is minimized for the parameters of this experiment. This is mainly because the structure is dominated by the cutoff behavior. The propagated APT has a very stable CEP which slips only $0.06 \pi$ between successive pulses near the center of the train. This also means that pulse-to-pulse energy fluctuations of the driving laser (which are typically $\sim 1 \%$ ) are negligible in this context.

In conclusion, we have demonstrated that the odd and even harmonics generated by a two color laser field can be synchronized to yield a train of attosecond pulses with a spacing of one full IR cycle. This synchronization is a single atom effect due to the modification of the electron dynamics caused by the presence of the strong second harmonic field. The method is simple and robust against fluctuations in the intensities and relative phase of the two driving fields. It also yields attosecond pulses with a stable $\mathrm{CEP}$, even though we use nonstabilized femtosecond driving fields.
APTs with one pulse per IR cycle have an immediate application in strong field ionization studies since they can be used to lock the time when electrons enter the continuum to a particular phase of an IR field. This eliminates the phase ambiguity inherent in using APTs with a half-cycle periodicity [16]. It allows for detailed studies of the dynamics of electrons that interact with their parent ion after ionization, the basis for numerous strong field processes. It has also recently been demonstrated that the individual pulses in an APT generated with odd harmonics can be compressed to the single cycle limit [12]. In the conditions that lead to phase locking in the present experiment, i.e., values of $\varphi$ for which the high $\rightarrow$ low evolution dominates the harmonic spectrum, the synchronization methods employed in [12] can be expected to work with equal efficacy. We have therefore demonstrated a clear route to the generation of CEP stabilized, few cycle pulses in the XUV wavelength region.

This research was supported by the Swedish Science Council, the National Science Foundation through Grants No. PHY-0401625 and No. PHY-0449235, the Integrated Initiative of Infrastructure LASERLAB-EUROPE (No. RII3-CT-2003-506350) within the 6th European Community Framework Programme, and the Knut and Alice Wallenberg Foundation.

[1] J. Reichert et al., Opt. Commun. 172, 59 (1999).

[2] T. Udem, R. Holzwarth, and T. W. Hänsch, Nature (London) 416, 233 (2002).

[3] E. Goulielmakis et al., Science 305, 1267 (2004).

[4] T. Brabec and F. Krausz, Rev. Mod. Phys. 72, 545 (2000).

[5] D. J. Jones et al., Science 288, 635 (2000).

[6] G. G. Paulus et al., Nature (London) 414, 182 (2001).

[7] A. Baltuška et al., Nature (London) 421, 611 (2003).

[8] G. G. Paulus et al., Phys. Rev. Lett. 91, 253004 (2003).

[9] F. Lindner et al., Phys. Rev. Lett. 95, 040401 (2005).

[10] P. M. Paul et al., Science 292, 1689 (2001).

[11] Y. Mairesse et al., Science 302, 1540 (2003).

[12] R. López-Martens et al., Phys. Rev. Lett. 94, 033001 (2005).

[13] M.D. Perry and J. K. Crane, Phys. Rev. A 48, R4051 (1993).

[14] H. Eichmann et al., Phys. Rev. A 51, R3414 (1995).

[15] I. J. Kim et al., Phys. Rev. Lett. 94, 243901 (2005).

[16] P. Johnsson et al., Phys. Rev. Lett. 95, 013001 (2005).

[17] J. Itatani et al., Phys. Rev. Lett. 88, 173903 (2002).

[18] The duration of the APT pulses cannot be directly obtained from this measurement since we collect electrons over a $2 \pi$ solid angle. We estimate it to be $\sim 300$ as.

[19] M. Lewenstein et al., Phys. Rev. A 49, 2117 (1994).

[20] P. Antoine, A. L'Huillier, and M. Lewenstein, Phys. Rev. Lett. 77, 1234 (1996).

[21] K. J. Schafer and K. C. Kulander, Phys. Rev. Lett. 78, 638 (1997).

[22] M. B. Gaarde et al., Phys. Rev. A 72, 013411 (2005).

[23] K. Varjú et al., Phys. Rev. Lett. 95, 243901 (2005). 